\title{
e-Phaïstos
}

e-Phaïstos

Revue d'histoire des techniques / Journal of the history

of technology

I-1 | 2012

Le patrimoine maritime

\section{Des mots et des techniques}

À propos de la construction navale vernaculaire tunisienne à la veille de la Seconde Guerre mondiale dans la région de Sfax et des îles Kerkenna

\section{Éric Rieth}

\section{(2) OpenEdition}

Journals

Édition électronique

URL : http://journals.openedition.org/ephaistos/208

DOI : 10.4000/ephaistos.208

ISSN : 2552-0741

Éditeur

IHMC - Institut d'histoire moderne et contemporaine (UMR 8066)

Édition imprimée

Date de publication : 1 juin 2012

Pagination : 12-19

ISSN : 2262-7340

Référence électronique

Éric Rieth, «Des mots et des techniques », e-Phaïstos [En ligne], I-1 | 2012, mis en ligne le 01 janvier 2015, consulté le 03 mai 2019. URL : http://journals.openedition.org/ephaistos/208 ; DOI : 10.4000/ ephaistos.208 


\title{
Des mots et des techniques. À propos de la construction navale vernaculaire tunisienne à la veille de la Seconde Guerre mondiale dans la région de Sfax et des îles Kerkenna
}

\author{
Éric Rieth \\ CNRS (LAMOP) \\ Musée national de la Marine
}

\begin{abstract}
Introduction
De quelle manière la terminologie participe-t-elle à l'écriture de l'histoire de l'architecture navale et, plus particulièrement dans le cadre de cet article, de quelle façon certains termes empruntés à l'italien et cohabitant avec des mots arabes contribuent-ils à l'histoire de l'architecture navale vernaculaire de la région de Sfax et des îles Kerkenna ? Cette interrogation s'inscrit dans le prolongement de la publication d'une enquête ethnographique réalisée par Jean Poujade en 1939 auprès de deux charpentiers de marine d'origine maltaise et italienne et demeurée jusqu'alors inédite ${ }^{1}$. Évoquant son enquête, J. Poujade écrivait :
\end{abstract}

\begin{abstract}
« Nous avons rencontré sur la plage de Sfax un Italien et un Maltais qui construisaient ensemble, près d'une cabane qui abritait quelques outils, une barque ; celle-ci était de forme grecque et destinée à des Tunisiens ; c'est un des plus beaux exemples de métissage des formes et des procédés que nous ayons rencontré au cours de nos croisières $»^{2}$.
\end{abstract}

C'est autour de cette notion de "métissage des formes et des procédés » que notre réflexion s'est orientée. Ce «métissage » des techniques de construction navale observée par Poujade se traduisait-il éga- lement en " métissage linguistique » ? Ce sont ces relations entre mots et techniques qui tissent le fil d'Ariane des pages qui vont suivre.

Pour développer cette réflexion, on dispose d'une assise remarquable: L'Atlas et le glossaire nautique tunisiens rédigé par Albert Gateau ${ }^{3}$. Agrégé d'arabe en 1933, A. Gateau a dirigé une part de ses recherches vers l'étude des sources arabes relatives à la conquête de l'Afrique du Nord et de l'Espagne et une autre part, toute aussi importante, vers l'étude de la vie maritime en Tunisie, sujet de sa thèse demeurée inachevée en raison de son décès prématuré. C'est au cours de son séjour à Tunis entre 1928 et 1934 comme professeur d'arabe au renommé collège Sadiki qu'il effectua l'essentiel de ses enquêtes ethno-linguistiques.

Cette recherche se déroule à une époque où la Tunisie, depuis le traité du Bardo signé en 1881 et complété en 1883 par la convention de La Marsa, était un protectorat français. Ce statut de protectorat, qui a duré jusqu'en 1956 et l'indépendance de la Tunisie, a été précédé par des relations diplomatiques et commerciales anciennes entre la France et la Tunisie. C'est ainsi que quelques années après la conquête turque 
(1574) est installé le premier consul français (1577). En 1665, un traité entre la Régence et la France accorde au consul de France la prééminence sur les autres consuls.

Comme l'indique le révérend père Charles, éditeur scientifique du livre (atlas et glossaire) d'A. Gateau,

\begin{abstract}
«... À plusieurs reprises, sur la Ouarda, « La Rose », ... [A. Gateau] partage la vie de marins, transporteurs de sable au long de la côte tunisienne. Il fait également plusieurs séjours à Jerba et rassemble ainsi peu à peu les matériaux linguistique et ethnographique de ses études sur la vie maritime en $\mathrm{Tu}-$ nisie $»^{4}$.
\end{abstract}

À l'apport, fondamental, de l'ouvrage d'A. Gateau vient s'ajouter le travail d'enquête ethno-historique de notre collègue tunisien de l'Université de Sfax Abdelhamid Barkaoui, spécialiste de l'histoire maritime et du patrimoine nautique de la Tunisie, et celui de nos propres études ethno-archéologiques sur l'architecture navale vernaculaire de la Méditerranée.

Au stade actuel d'avancement de nos recherches, cette étude est à considérer comme une première réflexion destinée à présenter des données et à formuler des interprétations sous forme avant tout d'hypothèses de travail susceptibles d'être corrigées, complétées, précisées.

Commençons par une première question concernant le gabarit appelé «stamenare ».

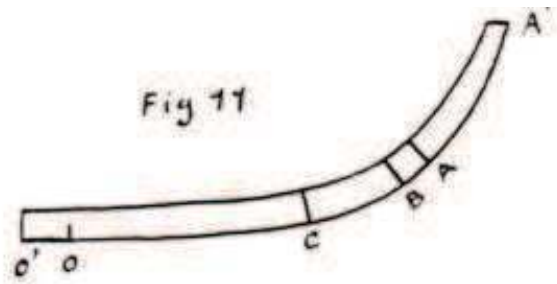

Fig. 1 : Le « stamenare » des charpentiers de Sfax (d'après J. Poujade, op. cit., fig. 11)

\section{La question du "stamenare"}

Dans son enquête menée à Sfax en 1939 auprès de deux charpentiers de marine, l'un d'origine italienne né à Sfax et l'autre d'origine maltaise et naturalisé français ${ }^{5}$, le seul terme étranger cité par J. Poujade est celui de "stamenare". Ce mot d'origine italienne est employé par les deux charpentiers pour désigner le maître-gabarit qui, d'une façon similaire au gabarit de Saint-Joseph provençal et languedocien décrit par Jules Vence en 1897 ,

« ... n'est autre que celui du maître-couple [et] sert, au moyen de certains repères, à gabarier la membrure sur une longueur [en pans de $0,25 \mathrm{~m}$ ] variant de 0,33 à 0,40 de la longueur totale du bateau au milieu. Les membres extrêmes avant et arrière sont cueillis [c'est-à-dire définis] en place, suivant des fausses lisses, comme des couples de remplissage $»^{6}$.

L'usage d'un tel terme n'est en rien exceptionnel au regard de l'origine des charpentiers. D'une façon plus générale, ce mot de "stamenare" renvoie à ce que le linguiste Jan Fennis appelle le « langage des galères »7, c'est-à-dire à cette langue technique méditerranéenne qui s'applique tout autant aux unités de la famille des galères qu'à celle des navires à voile de guerre, de commerce ainsi qu'à celle des bateaux de pêche. En revanche, le sens donné au mot "stamenare" par les charpentiers de marine de Sfax, à savoir le maître-gabarit ou gabarit de Saint-Joseph, ne correspond pas à la signification habituelle du terme.

Dans sa forme française de "stamenaire" ou "estamenaire" 8 , le mot désigne, en effet, non pas le gabarit mais l'allonge venant prolonger la varangue ou, pour reprendre la terminologie méditerra-

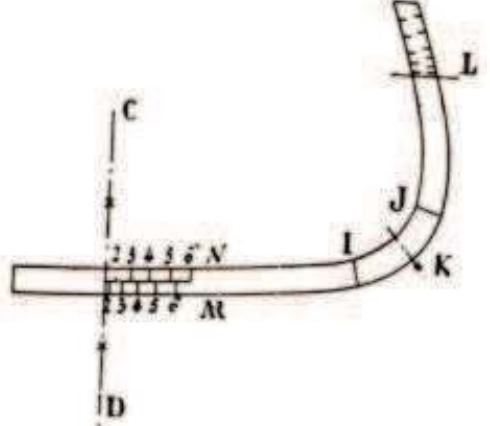

Fig. 2 : Le gabarit de Saint-Joseph (d'après J. Vence, op. cit., fig. 2) néenne, le "madier".

Première différence donc : le « stamenaire » du langage traditionnel correspond à une pièce de charpente et non à un gabarit ; deuxième différence : le "stamenare" " des charpentiers de Sfax correspond à une demi-membrure ${ }^{9}$ alors que le "stamenaire" comme pièce de charpente est limité à une partie de la membrure, l'allonge. Ajoutons une dernière précision. Sous sa forme de "estaminaire", la plus ancienne attestation en français du mot provenant d'un compte du Clos des galées de Rouen date de 1379: «... estaminairez, petis, 
grans madiers $»^{10}$. Sous sa forme latine, la plus ancienne mention connue extraite d'un compte de construction de galées près de Narbonne remonte aux années 1318-1320 : " ... pro 81 tallis de stamenarres $»^{11}$.

L'une des questions posées par le mot "stamenare" tel qu'il a été employé par les deux charpentiers de Sfax en 1939 est celle de sa place dans le vocabulaire nautique arabe de Tunisie.

L'ouvrage de référence en la matière, celui d'A. Gateau, ignore le terme. Dans son glossaire, l'allonge est dénommée "skarmo"12 et le gabarit "qâleb" il le rappeler, les enquêtes réalisées par A. Gateau se situent dans la décennie des années 1930 soit quelques années seulement avant les recherches effectuées par J. Poujade. Le contexte temporel est, par conséquent, très proche et ne parait pas pouvoir constituer une explication à la différence du vocabulaire. Dans ces conditions, l'interprétation la plus logique serait de considérer le mot "stamenare", dans le sens spécifique de gabarit, comme propre aux deux charpentiers de Sfax et à leur culture technique. Ajoutons que cette absence du terme "stamenare" dans le vocabulaire de la construction navale arabe tunisien est également présente de nos jours. C'est ainsi que les charpentiers de marine des îles Kerkenna situées au large de Sfax désignent les allonges sous le terme de "khétil" et le gabarit, l'équivalent du "stamenare", sous celui de "qualîb el qrabuss"14.

L'autre question soulevée par le mot "stamenare" est celle de la relation entre le mot - l'immatériel - et l'objet - le matériel - qu'il traduit. Comme nous l'examinerons ultérieurement, cette absence du mot "stamenare" ne signifie nullement une absence d'emploi de la méthode du "stamenare", c'est-à-dire du maître-gabarit. Celui-ci représente bien, en effet, l'un des "instruments de conception", avec la tablette d'acculement - "mîtra" -, particuliers à la construction navale vernaculaire tunisienne.

Avec la question du "stamenare" se dessine en fili- grane celle de la présence d'une terminologie d'origine italienne dans le vocabulaire usuel arabe tunisien de la construction navale.

\section{Des mots venus "d'ailleurs", de la rive nord de la Méditerranée}

Considérons quelques cas significatifs de cette présence du vocabulaire d'origine italienne. Au niveau de la charpente longitudinale de la coque, deux termes arabes sont utilisés, l'un pour qualifier la quille, "qrêna" -, l'autre pour désigner la carlingue - "kontra qrêna" -. Le premier mot est dérivé de l’italien "carina" ou "carena" et le second de l'italien ${ }^{15}$ "contra carina". Ces deux mots sont eux-mêmes dérivés du latin " $c a-$ rina/carena" dont la plus ancienne attestation est datée de 1253: « ... excepto quod quando carena navis videbitur prima die $»^{16}$. Ajoutons que dans la terminologie française moderne (jusqu'à la fin du XVIII ${ }^{\mathrm{e}}$ siècle) de l'architecture navale méditerranéenne, la quille est appelée "carène" et la carlingue "contre carène". Un troisième terme arabe de la charpente longitudinale est celui désignant l'étrave, "rûta" ${ }^{17}$ dont la forme italienne d'origine est "ruota", "rota" ou "roda". Les termes italiens dérivent du latin "rota/roda" dont la plus ancienne attestation remonte à $1268:$ " ... et habebit a triginta octo gois usque ad triginta novem goas de roda in roda $»^{18}$. Observons que dans le vocabulaire nautique méditerranéen contemporain, le terme se retrouve sous la forme française de "rode de proue" pour l'étrave et de "rode de poupe" pour l'étambot.

$\mathrm{Au}$ niveau de la charpente transversale, c'est-à-dire des membrures principalement, deux termes arabes sont à retenir. Le premier qualifie une membrure acculée et pincée des extrémités de la coque, - "forka" -19 dont l'origine italienne "forca" dérive directement, suivant une voie classique, du latin "forca" qui signifie une fourche. La forme française est celle de "fourcat", un terme toujours employé en France dans la construction navale en bois. Le deuxième mot est celui de "lâta" "2o qui désigne un bau ou un barrot appelé dans le langage 


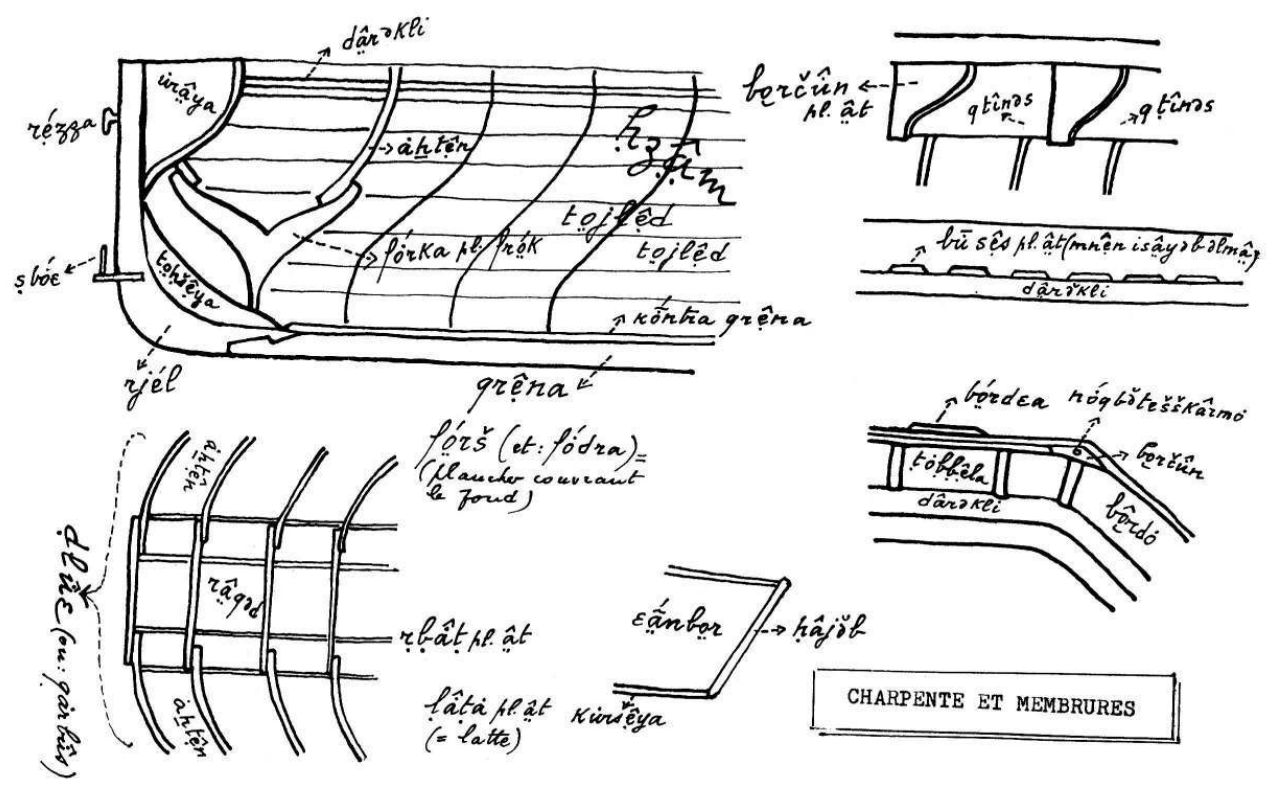

Fig. 3 : Les termes associés à la coque (d'après A. GATEAU, 1966, op. cit., vol. 1 pl. XXI)
D्ञ $\quad$ bulaire de la construction navale vernaculaire tunisienne, en particulier dans la région de Sfax et des îles Kerkenna ${ }^{25}$. Comment interpréter ces mots venus "d'ailleurs", de la rive nord de la Méditerranée ? Il faut tout d'abord souligner que ces termes d'origine italienne présents dans le vocabulaire nautique arabe tunisien sont nombreux ${ }^{26}$. En deuxième lieu, leur maintien actuel, plus de cinquante ans après l'indépendance de la Tunisie, semble révélateur de leur enracinement dans la culture technique des chantiers navals tuni- des galères de France une "latte" et dont la mention la plus ancienne date de 1550 : « Et pour la pouppe et proüe fault cinquante lattes au pris de huict solz pour piece ${ }^{21}$. Le mot dérive de l'italien "lata" qui lui-même provient du latin "lata” dont l'attestation la plus ancienne date de 1318-1320 : « ... pro vectura 310 latarum in dictis galeis necessarium ${ }^{22}$.

Quelques autres termes d'origine italienne sont également usités comme, par exemple, celui de "santîna" "23, qui provient de l'italien "sentina" lui-même dérivé du latin "sentina” et dont l'une des plus anciennes mentions date de 1298 : « ... qui Symon intravit ad sentenam gualee juanensis ${ }^{24}$. La forme française, guère différente, caractérisant le point le plus bas des fonds de la coque où se concentrent les eaux d'infiltration, est celle de "sentine".

Les termes "qrêna" ("qrîna"), "forka" ("furka"), "lata" ("lattat"), sont toujours employés dans le voca- siens, et kerkenniens en particulier. Ces termes originaires de la rive nord de la Méditerranée apparaissent avoir été totalement assimilés et arabisés pour devenir désormais des mots usuels du vocabulaire technique arabe de Tunisie. A cet égard, on peut se demander pour quelle raison une pièce de charpente aussi essentielle que la quille est désignée par un mot emprunté à la terminologie technique italienne et non par un terme arabe alors que la construction "sur quille" de principe "sur membrure première" devait être pratiquée, en toute probabilité, sur le territoire tunisien bien avant le début de la Régence turque, à l'époque des Fatimides ou des Hafsides.

En tout état de cause, on se trouve bien, semble-til, avec cet emploi des termes de "qrêna" ("qrîna"), "forka" ("furka"), "lata" ("lattat"), dans un contexte différent de celui évoqué à propos d'un autre mot venu "d'ailleurs", celui de "stamenare", que les deux 
charpentiers de marine de Sfax, l'un d'origine italienne, l'autre d'origine maltaise, employaient en 1939 pour qualifier le maitre-gabarit et dont l'usage ne semblerait pas avoir dépassé les limites étroites de leur chantier naval installé sur la plage pour construire une "flûka".

Comment peut-on essayer d'expliquer l'influence du vocabulaire d'origine italienne dans les pratiques linguistiques actuelles de la Tunisie ? Une partie de l'explication est à rechercher, sans doute, dans l'importance de l'émigration des pêcheurs italiens en Tunisie. Le commandant Hennique, dans son ouvrage publié en $1888^{27}$, mais dont les données statistiques, notamment, concernent la période précédant le protectorat de la France ${ }^{28}$, met bien en évidence une présence massive de la communauté italienne en Tunisie. Concernant la pêche des éponges qui représente une activité très importante ${ }^{29}$, il y avait ainsi en 1882 plus de 500 embarcations armées par des pêcheurs tunisiens. Cette même année 1882 considérée par Hennique “... comme une année moyenne", 200 "barchétas" ou "loudsos" armés par des pêcheurs siciliens ont participé à la campagne de pêche aux éponges puis ont regagné la Sicile avec leurs bateaux en fin de campagne pour 150 d'entre eux. À ces pêcheurs siciliens venus sur leurs propres bateaux s'ajoutent d'une part ceux ayant embarqué leurs "barchétas" sur des voiliers et d'autre part les pêcheurs grecs ${ }^{30}$ et, enfin, depuis les années 1880, les pêcheurs maltais de Sfax. Au total, ce sont plus de 350 bateaux armés par des pêcheurs siciliens, grecs et maltais qui, en 1882, ont participé à la campagne de pêche aux éponges soit près de $42 \%$ de la population totale de pêcheurs tunisiens et étrangers.

En 1891, 12000 marins et pêcheurs environ armaient 2500 bateaux dont 65\% étaient tunisiens, $30 \%$ italiens et $5 \%$ grecs et maltais ${ }^{31}$. Dix ans après le début du protectorat de la France, on constate, par conséquent, que l'importance des marins et pêcheurs italiens, siciliens majoritairement, demeure toujours aussi élevée. Ces chiffres semblent indiquer que le protectorat ne paraît avoir eu aucune conséquence sur la part de la population française dans les activités de pêche.
C'est, en effet, dans les activités agricoles, commerciales et industrielles d'une part et dans celles de la fonction publique et de l'armée d'autre part que la majorité de la population active française était impliquée.

Aux pêcheurs et marins italiens devaient s'ajouter, on peut le supposer, des charpentiers de marine qui, s'ils ne construisaient pas de bateaux, les réparaient et les calfataient ${ }^{32}$. Au regard de cette émigration italienne temporaire et, pour une part, permanente ${ }^{33}$, il semble logique de retrouver des mots du parler technique italien de la construction navale dans la terminologie nautique tunisienne. Mais le vocabulaire technique des charpentiers de marine tunisiens fait surtout appel à des mots arabes.

\section{Des mots venus “d'ici”, de la rive sud de la Mé- diterranée}

Deux termes liés à la charpente transversale ont été retenus en raison de leur importance dans l'histoire du développement de l'architecture navale méditerranéenne "sur membrure première" dont les origines datent des débuts de l'expansion arabo-musulmane dans les territoires de la rive sud de la Méditerranée.

Le premier mot qualifiant une varangue et, plus largement lorsqu'il s'agit d'une embarcation de taille modeste, l'ensemble de la membrure, varangue et allonges, est celui de "qorbûs" que l'on trouve aussi sous la forme de "qarbûs" 34 . Ce mot qui est toujours en usage pourrait renvoyer, nous semble-t-il, au terme italien "garbo"35 qui, dans la construction navale vernaculaire italienne, sarde, sicilienne, désigne le maître-gabarit, équivalent du gabarit de Saint-Joseph provençal et languedocien. Compte tenu de la proximité entre les deux termes - le q de "qarbûs" se transformant en g de "garbo"-, on peut se demander, à titre d'hypothèse de recherche, si l'étymologie de l'italien "garbo" ne se trouverait pas dans l'arabe "qarbûs". Dans cette hypothèse, le mot arabe associé à l'origine à la pièce de charpente ouvragée et finie - la varangue ou la totalité d'une membrure - serait devenu le mot italien correspondant à l'instrument de conception - le maître-gabarit - ayant servi à déterminer la forme 


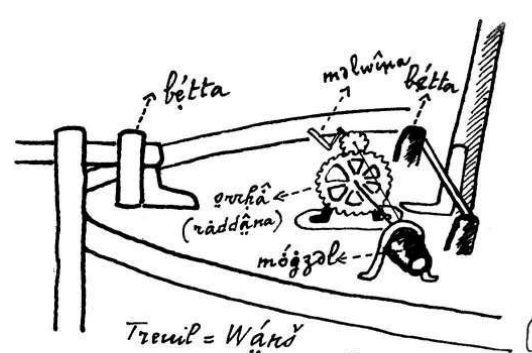

(Pli igâllac bẹth elmihtâf). mógzol (az. Zanzêl wuò śrma

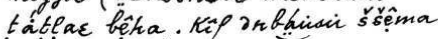
nrobtitia lelétta.)

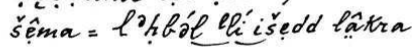
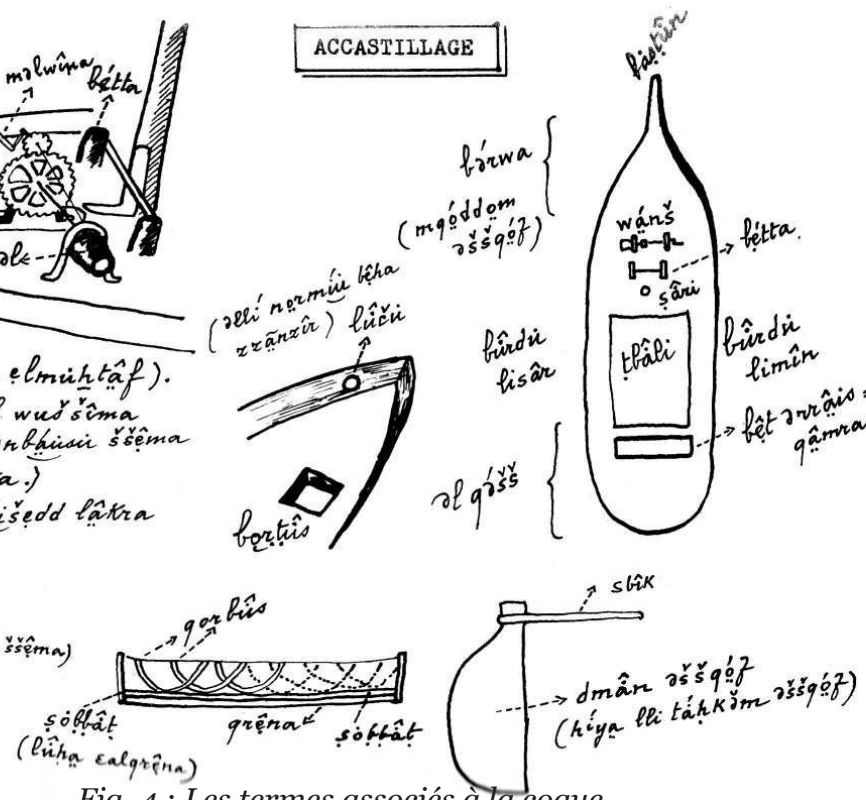

Fig. 4 : Les termes associés à ta coque

(d'après A. GATEAU, 1966, op. cit., vol. 1 pl. XXI)
Selon A. Gateau, « ... les formes gabari, prov. [çal] garbi, esp. [gnol], it. [talien] x garbo, galibo proviennent probablement toutes de l'arabe qalib ${ }^{40}$. Au regard de cette probable étymologie arabe, deux points sont à préciser. D'une part, la forme latine "galbe", "galebe", est attestée dès 1318-1320: "... clavellis parvulis ad clavandum mensuras tenues que vocantur galbe pro madayris galearum $»^{41}$. D'autre part, les formes espagnole "gàlibo" et surtout catalane "gàlib", "gàlip" 42 , sont très proches du terme arabe "qâlib".

Si la Catalogne conquise en 718 par les Arabo-musulmans est rapidement devenue une marche de l'Empire Carolin-

même de la pièce de charpente - varangue ou membrure - avant sa réalisation ${ }^{36}$. Le passage linguistique se serait ainsi effectué de la rive sud vers la rive nord de la Méditerranée. Ajoutons qu'il existe un mot français ancien - "garbe" - qui est à rapprocher des termes précédents, et qui sert à qualifier les formes de la coque d'un bâtiment. S'agissant par exemple d'une galère, il est ainsi mentionné dans un traité de construction de 1691 que « ... [l'] on donne [aux formes] ... le plus de longueur que l'on peut afin qu'elles obéissent mieux et qu'elles prennent bien le garbe de la galère ${ }^{37}$.

Le deuxième mot est celui désignant le gabarit utilisé pour prédéterminer la forme d'une partie des membrures, le "qâleb"38. Toujours usité, le terme de "qâleb" ou "qâlib" renvoie, dans le cadre de la construction navale vernaculaire kerkennienne spécialement ${ }^{39}$, au maître-gabarit qui, en relation avec la tablette d'acculement - "mîtra" -, fonctionne suivant un principe similaire à celui du gabarit de Saint-Joseph. gien, une partie importante de l'Espagne est demeurée sous la domination arabo-musulmane jusqu'à la prise

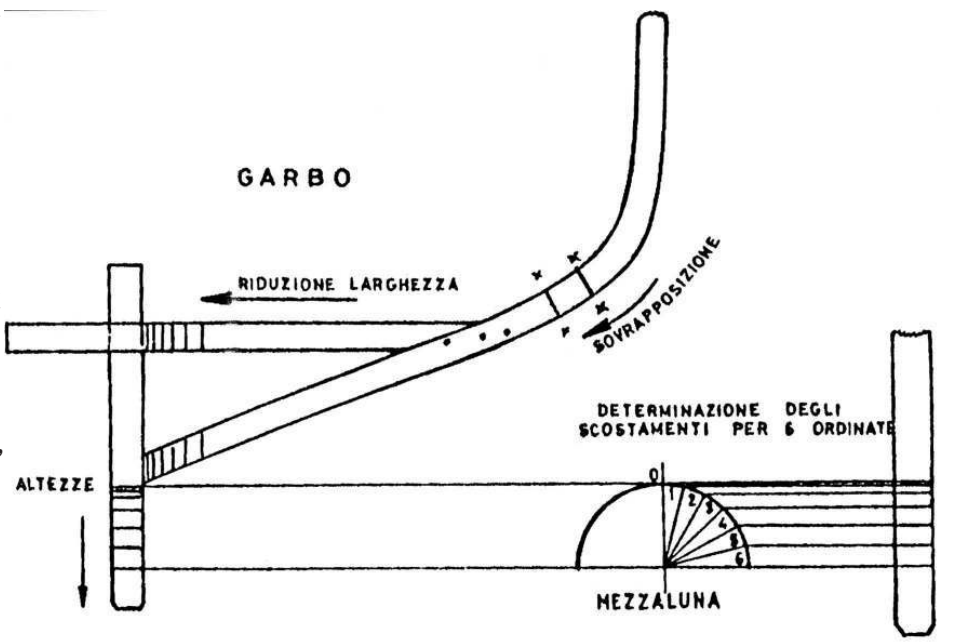

Fig. 5 : Le "garbo" du charpentier de marine sarde Pasquale Polese (d'après M. Marzari, 1979, op. cit., p. 204) 
de Grenade en 1492. À l'image de l'emprise de la culture arabo-musulmane sur les arts du monde ibérique - de l'architecture à la poésie et la musique -, il n'apparaît pas invraisemblable de supposer que cette même influence s'est également portée sur les techniques de la construction navale comme il en a été pour les techniques d'irrigation par exemple. Dans cette hypothèse, l'origine probablement arabe des termes "gàlibo", "gàlib" ou encore "gàlip" pourrait être interprétée, dans le contexte de l'histoire de l'architecture navale méditerranéenne du haut Moyen-Âge, comme un possible indice de l'introduction de la construction navale à franc-bord "sur membrure première" en territoire ibérique, corrélativement à l'expansion arabo-musulmane. Cette interprétation reposant sur des arguments linguistiques et qui, faut-il le rappeler, est à considérer comme une hypothèse de recherche, rejoindrait ainsi notre analyse, basée sur des données archéologiques, des origines différentes de l'architecture navale méditerranéenne "sur membrure première" dont l'une serait de tradition maritime gréco-romaine et l'autre de tradition fluvio-maritime nilotique ${ }^{43}$, antérieurement à la conquête de l'Egypte par les Arabo-musulmans en 613 ap. J.-C. Ce foyer nilotique d'architecture navale "sur membrure première" se serait ensuite développé vers l'ouest, vers le Maghreb, puis l'Espagne et le Portugal, suivant l'expansion arabo-musulmane.

\section{Conclusion}

Des mots et des techniques : l'étude de cette relation a essayé de mettre en évidence, à travers ce que J. Poujade appelait « ... un des plus beaux exemples de métissage des formes et des procédés [de construction] », un cas d'interpénétration des mots et des techniques entre les deux rives de la Méditerranée ${ }^{44}$. Cet exemple de "métissage" concernait, rappelons-le, la construction à Sfax, en 1939, d'une barque de tradition grecque pour des pêcheurs tunisiens par deux charpentiers d'origine italienne et maltaise. Au-delà de ce regard porté sur l'univers contemporain des techniques de construction navale méditerranéenne, cette étude a aussi tenté de rattacher cette relation entre des mots et des techniques à un passé plus lointain, celui de l'histoire des débuts de l'architecture navale "sur membrure première", une histoire dont l'élaboration repose sur les seuls vestiges archéologiques, subsituts des mots contemporains des charpentiers de marine tunisiens de la région de Sfax et des îles Kerkenna en particulier.

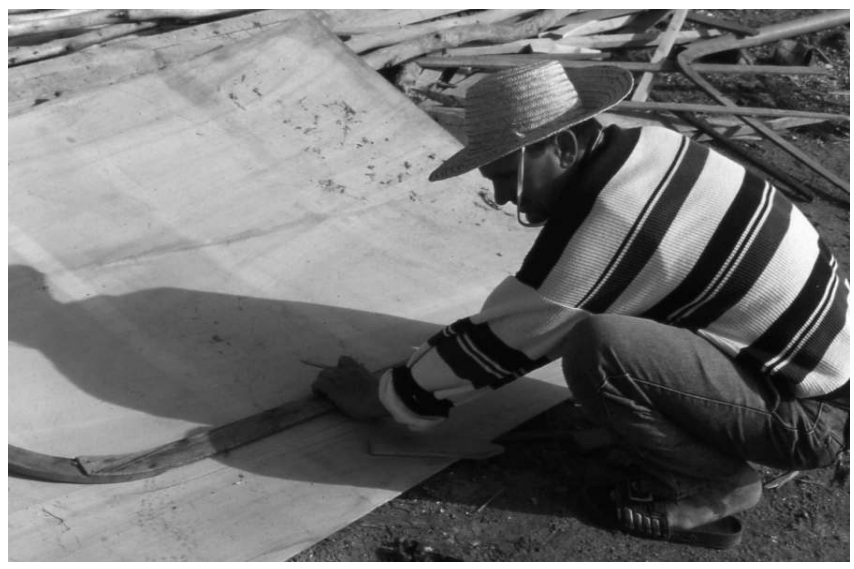

Fig. 6 : Chelly Zouheir, charpentier de marine à El Atä̈a, Kerkenna, trace une membrure avec un "qâleb" (photo : E. RIETH, CNRS) 
1 RIETH Éric, « Construction d'une barque de pêche à Sfax, Tunisie, en 1939 : une enquête inédite de Jean Poujade », Archeologia Maritima Mediterranea, 8, 2011, p. 153-177.

2 POUJADE Jean, La route des Indes et ses navires, Éditions Payot, Paris, 1946, p. 262.

3 GATEAU Albert, Atlas et Glossaire nautique tunisiens, édité par CHARLES Henri, Dar El-Machreq Éditeurs, Beyrouth, 1966, 2 vol.

4 GATEAU, 1966, op. cit., vol. 1, p. 13.

5 RIETH, 2011, op. cit., p. 171-172 (fac-similé du texte dactylographié de J. Poujade).

6 VENCE Jules, Construction et manouvre des bateaux et embarcations à voile latine, A. Challamel Éditeurs, Paris, 1897, p. 25 (réédition Laffitte Reprints, Marseille, 1980).

7 FENNIS Jan, Trésor du langage des galères, Niemeyer, Tübingen, 1995, 3 vol.

8 Il en est de même de la forme italienne.

9 Plus exactement, le "stamenare" comme gabarit, se compose d'une demi-varangue et d'une allonge.

10 FENNIS, 1995, op.cit., vol. 2, p. 85 .

11 FOURQUIN Noël, RIGAUD Philippe, De la nave au pointu. Glossaire nautique de la langue d'oc (Provence, Languedoc). Des origines à nos jours, Objectifs Mer et Capian Méditerranée, Toulon-Saint-Tropez, 1993, p. 158.

12 GATEAU, 1966, op. cit., vol. 2, p. 158.

13 GATEAU, 1966, op. cit., vol. 2, p. 116.

14 BARKAOUI Abdelhamid, "Techniques et traditions navales aux îles Kerkenna, Tunisie. Enquête-collecte ». Cette étude encore inédite constitue l'un des chapitres d'un livre à paraître (BARKAOUI A., RIETH É.) sur l'architecture navale vernaculaire des îles Kerkenna. L'ouvrage donnera lieu à une édition bilingue arabe-français.

15 GATEAU, 1966, op. cit., vol. 1, p.l. XXI, XXIV, vol. 2, p. 125.

16 FOURQUIN, RIGAUD, 1993, op. cit., p. 89.

17 GATEAU, 1966, op. cit., vol. 2, p. 131.

18 FOURQUIN, RIGAUD, 1993, op. cit., p. 325.

19 GATEAU, 1966, op. cit., vol. 1, pl . XXI, vol. 2, p. 48.

20 GATEAU, 1966, op. cit., vol. I, pl. XXI, vol. 2, p. 87.

21 FOURQUIN, RIGAUD, 1993, op. cit., p. 222.

22 Ibidem

23 GATEAU, 1966, op. cit., vol. 1, pl. XXII, XXIII, vol. 2, p. 143.

24 FOURQUIN, RIGAUD, 1993, op. cit., p. 339.

25 Cf. BARKAOUI, à paraître.

26 Sur un test portant sur 128 termes, soit de $a d a b$ (droits de douane) à brîk (brick), 56 termes sont d'origine arabe, 5 d'origine italienne, 12 d'origine française, 4 d'origine latine, 4 d'origine grecque et 1 d'origine maltaise (GATEAU, 1966, op. cit., vol. 2, p. 2-31. Merci à Richard Barker de nous avoir fait part de ce test).

27 HENNIQUE P. A., Les caboteurs et pêcheurs de la côte de Tunisie, Gauthier-Villars et Fils, Paris, 1888 (réédition Oméga, Nice, 1999).
28 Il s'agit des premières données statistiques publiées portant sur les activités de pêche et de cabotage en Tunisie.

29 HENNIQUE, 1888, op. cit., p. 75-76.

30 La spécialité des grecs était la pêche aux éponges en scaphandre lourd à casque.

31 GATEAU, 1966, op. cit., vol. 1, pl. LVII bis, note 2.

32 A. Gateau note qu'à Sfax, des lanches, felouques et loudes étaient construits. Et il ajoute : « Ce sont des italiens qui calfatent et assurent les réparations ». En revanche, il ne fait aucune référence à une activité de construction qui, semblerait-il, se trouvait alors principalement exercée par des constructeurs tunisiens. Dans cette hypothèse se pose la question du caractère exceptionnel ou non du chantier naval décrit par J. Poujade dans son enquête de 1939 à Sfax.

33 Le gouvernement italien favorisa cette émigration avant le début du protectorat.

34 GATEAU, 1966, op. cit., vol. 1, pl. XXV, XXVI, vol 2, p. 121.

35 MARZARI Mario, « Evolution of shipbuilding and methodologies in Adriatic and Tyrrhenian traditional shipyards ", dans RIETH Éric (dir.), Concevoir et construire les navires. De la trière au picoteux, (Technologies, Idéologies, Pratiques, XIII, 1), Éditions Erès, Ramonville Saint-Agnès, 1979, p. 181-215.

36 Le terme italien pour désigner une membrure est "corba".

37 FOURQUIN, RIGAUD, 1993, op. cit., 188.

38 GATEAU, 1966, op. cit., vol. 2, p. 116.

39 BARKAOUI, à paraître.

40 GATEAU, 1966, op. cit., vol. 2, p. 116.

41 FOURQUIN, RIGAUD, 1966, op. cit., p. 188.

42 « Lettre G» dans Nouveau Glossaire Nautique d'Augustin Jal. Révision de l'édition de 1848. Éditions du CNRS, Paris, 1989, p. 712.

43 RIETH Éric, « Géométrie des formes de carène et construction « sur membrure première » (Ve-XIIe siècles). Une autre approche de l'histoire de la construction navale Méditerranéenne au Moyen-Âge», Archaeologia Maritima Mediterranea, 5, 2008, p. $45-68$

44 Nous formulons le souhait que cette question soit soumise à la critique philologique. 